\title{
Wavelet Design for Automatic Real-Time Eye Blink Detection and Recognition in EEG Signals
}

\author{
M. Miranda, R. Salinas, U. Raff, O. Magna
}

\section{Michael Miranda*}

Department of Informatic Engineering

Metropolitan University of Technology, Chile

Jose Pedro Alessandri 1242, Nunoa, Santiago, Chile

*Corresponding author: michael.miranda@utem.cl

\section{Renato Salinas}

Department of Mechanical Engineering

University of Santiago, Chile

Av. Libertador Bernardo O’Higgins 3363, Santiago, Chile.

renato.salinas@usach.cl

\section{Ulrich Raff}

Department of Physics

University of Santiago, Chile

Av. Libertador Bernardo O'Higgins 3363, Santiago, Chile.

ulrich.raff@usach.cl

\section{Oscar Magna}

Department of Informatic Engineering

Metropolitan University of Technology, Chile

Jose Pedro Alessandri 1242, Nunoa, Santiago, Chile

omagna@utem.cl

\begin{abstract}
The blinking of an eye can be detected in electroencephalographic (EEG) recordings and can be understood as a useful control signal in some information processing tasks. The detection of a specific pattern associated with the blinking of an eye in real time using EEG signals of a single channel has been analyzed. This study considers both theoretical and practical principles enabling the design and implementation of a system capable of precise real-time detection of eye blinks within the EEG signal. This signal or pattern is subject to considerable scale changes and multiple incidences. In our proposed approach, a new wavelet was designed to improve the detection and localization of the eye blinking signal. The detection of multiple occurrences of the blinking perturbation in the recordings performed in real-time operation is achieved with a window giving a time-limited projection of an ongoing analysis of the sampled EEG signal.

Keywords: Biological signals, electroencephalogram, brain computer interface, eye blink detection, pattern recognition, wavelet design.
\end{abstract}

\section{Introduction}

The electroencephalogram was designed to record the brain activity of a living being, specifically by sensors, with which electrical signals from the brain are captured. Any activity that does not come from the brain, that is, noise, is called an artifact. Depending on the origin, they can be divided into physiological and extra-physiological artifacts. The first ones are generated by the patient, on the other hand, extra-physiological artifacts arise from sources external to the individual, that is to say from the environment. Blinking of eyes is considered an ocular artifact, which is captured in the frontal zones Fp1-Fp2 of the international system 10-20 [10]. 
The eyeball acts as a dipole, with a positive pole oriented anteriorly (cornea) and a negative pole oriented posteriorly (retina). A blinking of eyes causes the positive pole to approach the fronto-polar electrodes Fp1-Fp2, producing symmetrical descending deflections [15]. The EEG records are manifested with periodic and unpredictable oscillations, having a greater spectral amplitude in certain frequency bands, which are divided into: $0.5-4$ Hertz (delta band), 4-8 Hertz (theta band), 8-12 Hertz (alpha band), 12 - 30 Hertz (beta band) and greater than 30 Hertz (gamma band) [4].

The extraction of characteristics is an important process in the classification of EEG signals, and additionally, it is also possible to relate patterns with a defined intentionality on the part of the individual. However, the relationship between the electrical signal and the intention is diffuse, besides it is necessary to filter the artifacts, to obtain a signal without noise, so as not to disturb the results. One of the artifacts that must be filtered is the eye blinking, but before filtering it must be detected. The blinking of eyes could be considered, in itself, a control signal, since in general an individual can generate voluntary blinks, being able to increase the frequency and / or amplitude, which in turn can be associated with machine instructions for a computer. From the previous conceptualization, it is possible to devise a brain-machine interface or BCI, which uses eye blinking as support data. A brain-machine interface or BCI, is defined as the technology that allows capturing brain waves to be processed by a computer with the intention of obtaining information about a state or cognitive process of the person. For example, in Dzitac et al. [7] changes in amplitude are studied in the different frequency bands, specifically the events of desynchronization (or event-related ERD) and their application to BCI.

The work that will be presented in this publication, shows the research results related to the automatic detection of eye blinking by designing an ad-hoc mother wavelet, which allows the analysis in the time domain.

\section{Materials and methods}

\subsection{Creation of an EEG signal database}

The design of a mother wavelet requires experimental data and validation, from which it is possible to extract the representative pattern present in the study signal. For this reason, a free access database of EEG signals was consulted to obtain experimental data. Subsequently, a software based on Matlab and Java was developed for obtaining in real time experimental validation samples, recording the EEG signal and the video of the face of the individual with the same time-base, this way to correlate the video with the EEG signal and clearly define the occurrence of the blinks.

\subsection{Obtaining experimental data}

To verify the main hypotheses of this work, the available data is located in www.physionet.org, specifically the PhysioBank database. This is a database of high-growth physiological digital signals, with good foundation in data related to the biomedical research community. Polysomnographic record signals, of multiple parameters, are included in this database including cardiopulmonary, neural and other fields of biomedicine. The records correspond to both healthy individuals and patients with a wide variety of conditions that concern different pathological implications, such as sudden cardiac death, failure congestive heart, epilepsy, motor disorders, sleep apnea and senility. This database is freely accessible via web and tends to cooperative activities, that is providing data for research and requesting the submission of results for its feedback. The PhysioBank collections are organized in more than 50 databases, each with a number of records, and 
each record contains information collected from a single subject.

\subsection{Obtaining validation data}

Validation data were obtained from a continuous EEG measurement with the biosensor consisting of a band designed by Neurosky MindWave TM, being one of the first to enter the market of EEG amplifiers for use in non-invasive BCI. Mindwave is very economical due to the simplicity that characterizes it, having one electrode in position FP1 and has the option of being compatible with both iOs and Android [13].
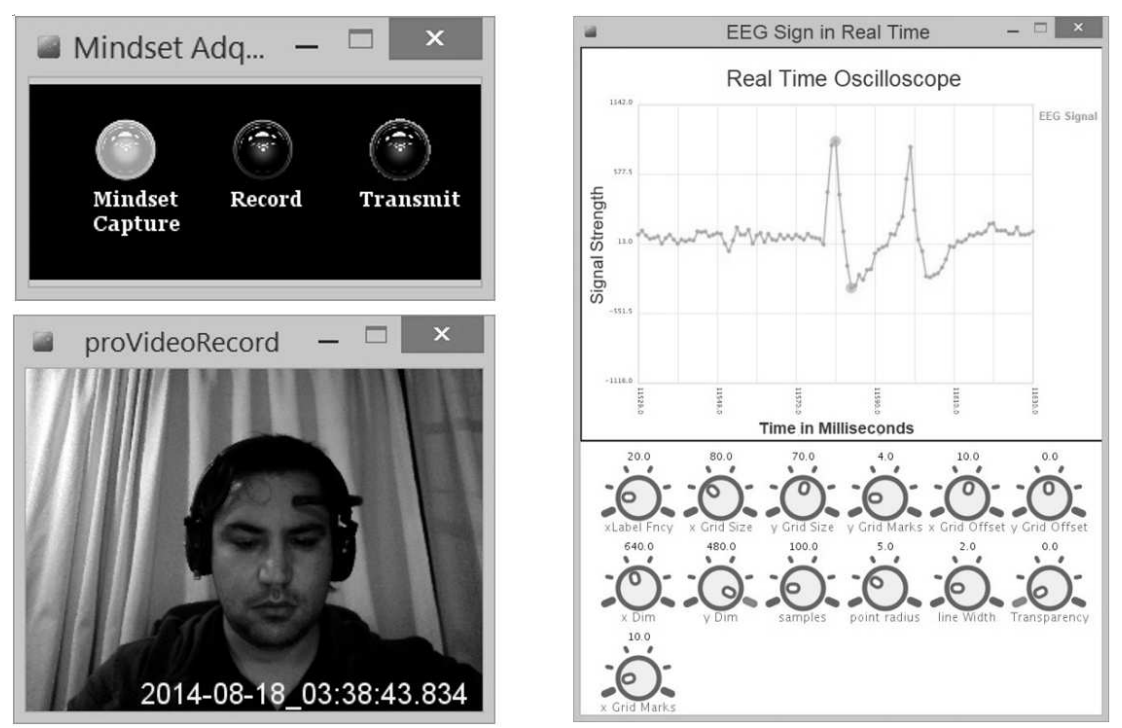

Figure 1: Video recording module, status indicator module and real-time software-oscilloscope module

The state of the eye blink was detected through a video camera during the EEG measurement, subsequently and then manually added to the file after analyzing the video frames. Where "1" indicates the closed eye state and "0" the state of the eye open. All the values are in chronological order with the first value measured in the top of the data. The platform developed is called "EEG Studio" and is composed of three modules:

1. Oscilloscope that works in real time.

2. Video recording: records the face of the individual in real time.

3. Status Indicator: indicates the correct functioning of the different modules.

\section{Wavelet transform}

Wavelets are functions that satisfy certain mathematical requirements and are used for the representation of data or other functions. Wavelets are very suitable for data approximation of signals with abrupt discontinuities. The fundamental idea behind wavelets is to analyze functions according to scales. In wavelet analysis, the scale used to analyze the data plays a special role. Algorithms that use wavelets process data at different scales or resolutions. If a signal or function is observed using a wide "window", the small details are not observed; On the other hand, if the "window" used is narrow, then they can be observed. In wavelet analysis, 
these windows are automatically adjusted when changing resolution, usually referred to as multiresolution analysis. This makes wavelets a useful and interesting tool. The general procedure of the analysis using wavelets is to adopt a "prototype" function, generally called mother wavelet. The temporal analysis is then carried out using dilatations and translations of said function. The original signal can then be represented as a linear combination of the original function and its translated and dilated ones. This procedure is called a wavelet expansion. The choice of the mother wavelet [13] (and thus the base or the wavelet frame) is not unique and depends on the type of functions or data to analyze. The multi-resolution analysis of the wavelets, makes it a very powerful tool for the study of EEG signals. An example of the EEG artifact and pattern of blinking of eyes is seen in Fig. 2.

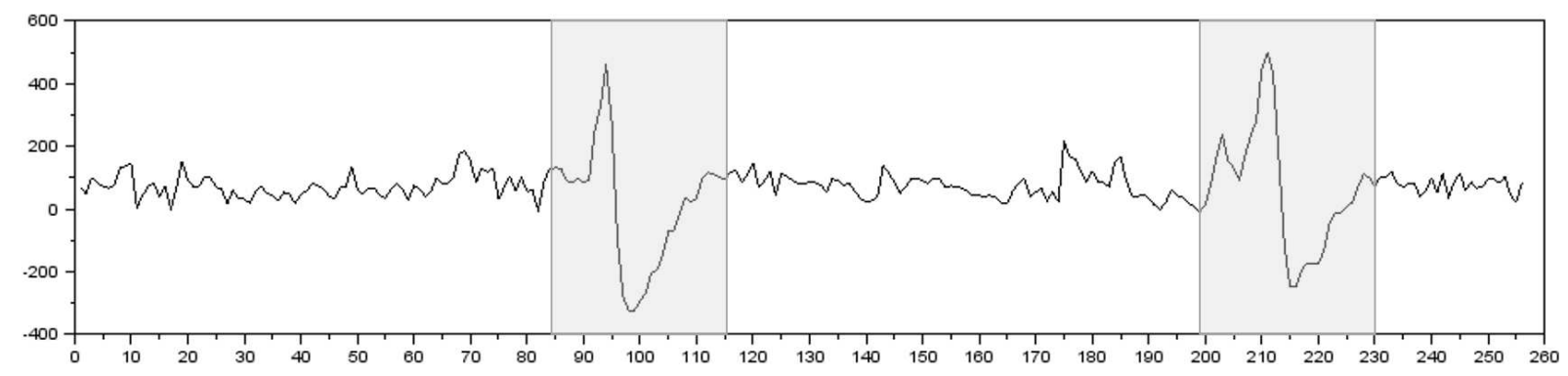

Figure 2: EEG signal with two artifacts produced by blinking eyes. The artifacts are indicated with two light gray time intervals

\subsection{Continuous wavelet transform}

The continuous Wavelet transform allows the analysis of a signal in a segment located in it and consists in expressing a continuous signal as an expansion of terms or coefficients of the internal product between the signal and a Mother Wavelet function. A Mother Wavelet is a localized function, belonging to space $L^{2}(R)$, that contains all functions with finite energy and square integrable functions defined:

$$
f \in L^{2} \Rightarrow \int|f(t)|^{2} d t=E<\infty
$$

In this way we have a single modulated window and from this a complete family of elementary functions is generated by dilatations or contractions and translations in time $\Psi_{a, b}(t)$, called wavelet daughters that meet all the conditions of the form:

$$
\Psi_{a, b}(t)=\frac{1}{\sqrt{a}} \Psi\left(\frac{t-b}{a}\right) \quad(a, b) \epsilon R, a \neq 0 .
$$

The Mother Wavelet must comply with the eligibility condition:

$$
C_{\Psi}(t)=\int_{0}^{\infty} \frac{|\Psi(\omega)|^{2}}{\omega} d \omega<\infty
$$

Which means that the function is well localized in time, that is, the function oscillates around an axis and its average is zero and that the Fourier transform is a continuous band-pass filter, with rapid decrease towards infinity and toward $\omega=0$. The Wavelet transform of a function at a given scale and position, is calculated by the correlation of the form: 


$$
C W T(a, b)=\frac{1}{\sqrt{a}} \int_{-\infty}^{\infty} x(t) \Psi\left(\frac{t-b}{a}\right) d t
$$

\subsection{Wavelet pattern design applied in $C W T$}

When searching a defined pattern it is possible to apply two different techniques to perform multiresolution analysis. The first technique includes the use of an existent wavelet family trying to find whatever fits best the detection of the pattern, i.e. trial and error technique [16]. In reference [16] the biorthogonal wavelet was used. The other alternative consists in designing or building a wavelet starting with the pattern that is asked to be detected. This process can obviously decrease the time to approach the desired goal while improving the detection of the blinking artifact. This approach will work as long as the characteristics of the designed wavelet are close enough to the characteristics of the recorded pattern. Based on a given function $f$ with compact support and finite energy, we may consider the construction of a wavelet usable with CWT while approximating this function in the least square sense. Various construction methods are possible, and since we have numerical sampling of our pattern over a given interval $[a, b]$, we used the method described by Misiti [14]. Let us consider a finite set of values:

$$
\left(t_{k}, y_{k}\right)_{k=1, \ldots, K} \quad, \text { such that }: a \leq t_{k} \leq b \text { and } y_{k} \approx f\left(t_{k}\right)
$$

Consider a family $F=\rho_{i=1}^{N}$ of linearly independent functions in $L^{2}(a, b)$, where $L^{2}$ is the space of square integrable functions over $\mathbb{R}$, and denote by $V$ the vector space spanning $F$. Formulated for this finite set of pairs, the problem consists of seeking coefficients $\alpha=\alpha_{i=1}^{N}$ in $\mathbb{R}^{N}$, where:

$$
\psi=\sum_{i=1}^{N} \alpha_{i} \rho_{i}
$$

such that:

$$
\sum_{k=1}^{K}\left[\psi\left(t_{k}\right)-y_{k}\right]^{2}=\underset{\beta \in \mathbb{R}^{N}}{\operatorname{Min}}\left\{\sum_{k=1}^{K}\left[v_{\beta}\left(t_{k}\right)-y_{k}\right]^{2}\right\}
$$

such that:

$$
\int_{a}^{b} v_{\beta}(t) d t=0
$$

where for $\beta$ in $\mathbb{R}^{N}$ :

$$
v_{\beta}=\sum_{i=1}^{N} \beta_{i} \rho_{i}
$$

It is thus a problem of least squares sense minimization with a constraint. The vector $\alpha$ and the Lagrange multiplier $\lambda$ associated with the constraint are obtained by solving the linear system:

$$
\left[\begin{array}{cc}
G & M^{t} \\
M & 0
\end{array}\right]\left[\begin{array}{c}
\alpha \\
\lambda
\end{array}\right]=\left[\begin{array}{c}
B \\
0
\end{array}\right]
$$

with $G, M$ and $B$ defined by:

$$
G_{i, j}=\sum_{k=1}^{K} \rho_{i}\left(t_{k}\right) \cdot \rho_{j}\left(t_{k}\right)
$$




$$
\begin{gathered}
M_{i}=\int_{a}^{b} \rho_{i}(t) d t \\
B_{i}=\sum_{k=1}^{K} y_{k} \cdot \rho_{j}\left(t_{k}\right)
\end{gathered}
$$

The wavelet $\psi(t)$, must satisfy conditions of admissibility and regularity. Regularity is defined as the capability of a given wavelet to reconstruct a signal from the coefficients computed during the transformation process [11]. In other words, a function is regular if it can be locally approximated by a polynomial. According to Lipschitz's definition of regularity [12], a function $\mathrm{f}$ is pointwise Lipschitz $\alpha \geq 0$ at $v$, if there exist $C>0$, and a polynomial $\rho_{v}$ of degree $m=[\alpha]$, such that:

$$
\forall t \in \mathbb{R}, \quad\left|f(t)-\rho_{v}(t)\right| \leq C \cdot|t-v|^{\alpha}
$$

A function $\mathrm{f}$ is uniformely Lipschitz $\alpha$ over $[a, b]$ if it satisfies for all $v \epsilon[a, b]$, with a constant $C$ that is independent of $v$. The Lipschitz regularity of $f$ at $v$ or over $[a, b]$ is the supremum of the $\alpha$ such that $f$ is Lipschitz $\alpha$. On the other hand, admissibility [11] is defined by the following conditions:

$$
\begin{gathered}
\int_{-\infty}^{\infty} \psi(t) d t=0 \\
\int_{-\infty}^{\infty}|\psi(t)|^{2} d t<\infty
\end{gathered}
$$

That is, function $\psi(t)$ must be localized in a bounded time interval, having oscillations around time axis, so its average be zero.

\subsection{Characteristic pattern detection}

Given the waveform pattern associated with eye blinking shown in Figure 2, it is necessary to design a mother wavelet as close as possible to the recorded pattern. This new wavelet will be labeled "blinkwave". This designed wavelet can then be used in the $C W T$ analysis because it fulfills the required wavelet properties using the $C W T$ technique [6].

\section{Design and use of the mother wavelet}

To design the new wavelet ("blinkwave") it becomes necessary to isolate the EEG Eye Blinking Pattern. Using this procedure, we obtain a vector or a set of finite number of values in equation (6). This vector is utilized in the process described by Misiti [14]. Designing the wavelet process with polynomials of variable grades, we obtain a mother wavelet with best approximation to the recorded pattern with a polynomial of grade 6, which can be observed in Figure 3.

In this case, the family $F=\rho_{i} N$ used in equations (5) to (13), is the polynomial family of grade $N \geq 6$. 


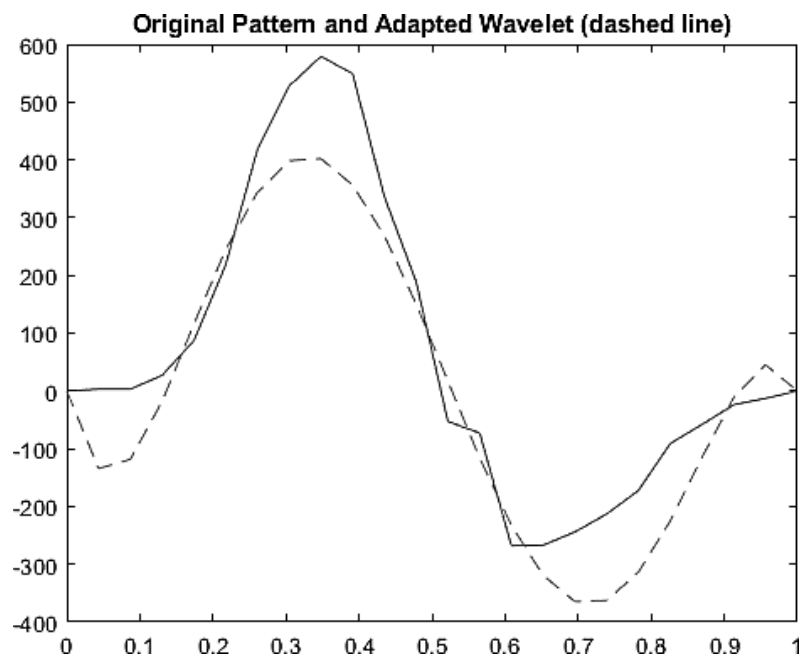

Figure 3: Overlay of the waveform produced by eye blinking and the wavelet designed, both normalized in the range $[-1,1]$

\subsection{Design of the mother wavelet}

According to the method hinted in [18], it is necessary to construct matrices $G, M$ and $B$, to fulfill equation (10), and to resolve the following linear system:

$$
\left(\begin{array}{cc}
G & M^{t} \\
M & 0
\end{array}\right)\left(\begin{array}{c}
\alpha \\
\lambda
\end{array}\right)=\left(\begin{array}{c}
B \\
0
\end{array}\right) \quad \Rightarrow \quad A \cdot U=B
$$

I.e., solve $U$ as a function of $A$ :

$$
U=A^{-1} \cdot B
$$

We take as base the pattern ("blinkwave"), which is defined as an n-component vector:

$$
\left\{y_{i}\right\}_{i=1}^{m}=y_{1}, y_{2}, \ldots, y_{m}
$$

The base pattern is obtained digitizing the continuous signal with a constant sampling frequency $f_{s}$ which defines the time interval $\Delta t$ between samples. The matrix or file vector $\mathrm{M}$, is built using the formula in equation (12), considering the admissibility restrictions given by equations (15) and (16), resulting in the following series:

$$
\begin{aligned}
& M_{i=[1, m]}=\int_{a}^{b} \rho_{i}(t) d t=\left\{\frac{1}{i}\left(b^{i}-a^{i}\right)\right\}_{i=[1, m]}= \\
= & {\left[(b-a), \quad\left(\frac{b^{2}}{2}-\frac{a^{2}}{2}\right), \quad \ldots,\left(\frac{b^{N+1}}{N+1}-\frac{a^{N+1}}{N+1}\right)\right] }
\end{aligned}
$$

If one chooses initiating the analysis at the origin, $a=0$, then all computations become simpler, then, recomputing $M$ we obtain:

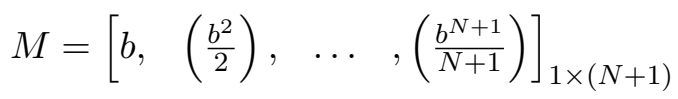

Given $M$, we must apply the regularity condition indicated in equation (14), to get a new matrix $M$, as follows: 


$$
M=\left[\begin{array}{cccc}
b, & \left(\frac{b^{2}}{2}\right), & \ldots & ,\left(\frac{b^{N+1}}{N+1}\right) \\
1 & 0 & \ldots & 0 \\
1 & b & \ldots & b^{N}
\end{array}\right]_{3 \times(N+1)}
$$

Afterwards, the matrix $G$ is built using equation (11):

$$
G=\left[\begin{array}{cccc}
b & \left(\frac{b^{2}}{2}\right) & \cdots & \left(\frac{b^{N+1}}{N+1}\right) \\
\left(\frac{b^{2}}{2}\right) & \left(\frac{b^{3}}{3}\right) & \ddots & \left(\frac{b^{N+2}}{N+2}\right) \\
\vdots & \vdots & \cdots & \vdots \\
\left(\frac{b^{N+1}}{N+1}\right) & \left(\frac{b^{N+2}}{N+2}\right) & \cdots & \left(\frac{b^{2 N+1}}{N+2}\right)
\end{array}\right]_{(N+1) \times(N+1)}
$$

Prior to solving the linear system for $U$, we must generate the column vector $B$, as described in equation (13):

$$
B=\left(\frac{1}{2}\right)\left[\begin{array}{c}
\sum_{i=1}^{m}\left(x_{i+1}-x_{i}\right)\left(x_{i+1} \cdot y_{i+1}+x_{i} \cdot y_{i}\right) \\
\sum_{i=1}^{m}\left(x_{i+1}-x_{i}\right)\left(x_{i+1}^{2} \cdot y_{i+1}+x_{i}{ }^{2} \cdot y_{i}\right) \\
\vdots \\
\sum_{i=1}^{m}\left(x_{i+1}-x_{i}\right)\left(x_{i+1}^{N} \cdot y_{i+1}+x_{i}{ }^{N} \cdot y_{i}\right)
\end{array}\right]_{(N+1) \times 1}
$$

Once matrices $G$ and $M$ are generated, it is possible assembling matrix $A$ and proceed solving equation (17):

$$
U=A^{-1} \cdot B \quad \Rightarrow \quad\left[\begin{array}{c}
\alpha_{1} \\
\alpha_{2} \\
\vdots \\
\lambda_{1}
\end{array}\right]=\left[\begin{array}{cc}
G & M^{t} \\
M & 0
\end{array}\right]^{11} \cdot[B]
$$

Mother wavelet $\psi(t)$, is generated using formula in equation (6) replacing the values obtained for $\alpha_{i}$

$$
\psi=\sum_{i=1}^{N} \alpha_{i} \cdot \rho_{i} \quad \Rightarrow \quad \psi(t)=\sum_{i=1}^{N+1} \alpha_{i} \cdot x_{i}{ }^{N+1-i}
$$

Thus, for the numerical pattern shown in Figure 3 (solid line), represented by vector:

$$
y=[0.0,1.7,2.0,1.6,0.8,0.0,-0.6,-0.9,-1.1,-1.0,-0.9,-0.7,-0.5,-0.4,-0.3,0.0]
$$

We obtain the polynomial mother wavelet $\psi(t)$ as follow:

$$
\psi(t)=\alpha_{1} \cdot x^{n}+\alpha_{2} \cdot x^{n-1}+\ldots+\alpha_{n} \cdot x^{n}+\alpha_{n+1}
$$

I.e., the first $\mathrm{n}$ numerical coefficients $\alpha_{i}$ are employed, obtained from solving equation (25) and discarding the Lagrange multipliers $\lambda_{i}$. These coefficients for the mother wavelet $\psi(t)$, for the pattern given in equation (27), are shown in equation (29) as follows:

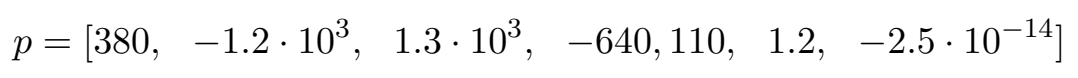


Finally, the polynomial equation for the new mother wavelet $\psi(t)$, is shown graphically in Figure 3 (dashed line) and it is represented algebraically by equation (30), as follows:

$$
\psi(t)=380 \cdot x^{6}-1200 \cdot x^{5}+1300 \cdot x^{4}-640 \cdot x^{3}+110 \cdot x^{2}+1.2 \cdot x-02.5 \cdot 10^{-14}
$$

\subsection{Use of the mother wavelet}

The new wavelet-blinkwave is used to obtain the coefficients, which will subsequently be processed to obtain the location of each artifact. Using the signal shown in Figure 3 or the "blinkwave" mother wavelet for analysis and applying the $C W T$, we obtain a coefficient matrix $C(a, b)$ using equation (31), where:

$$
C(a, b)=C W T(\text { "inputsignal","blinkwave") }
$$

$C(a, b)$ is the coefficients matrix and $C W T$ is the continuous wavelet transform, applied to input signal. The next step includes the detection and duration of the eye blinking from $C(a, b)$. To improve the analysis, a threshold must be applied to the matrix of wavelet coefficients, as defined in equation (32).

$$
T(a, b)=\left\{\begin{array}{ccc}
0 & \text { if } & C(a, b)<\text { Threshold }_{\text {fixed }} \\
C(a, b) & , & \text { otherwise }
\end{array}\right.
$$

The dimensions of the matrix depend on the number of samples of the analyzed signal and the level or number of scales in the decomposition performed by the wavelet transform.

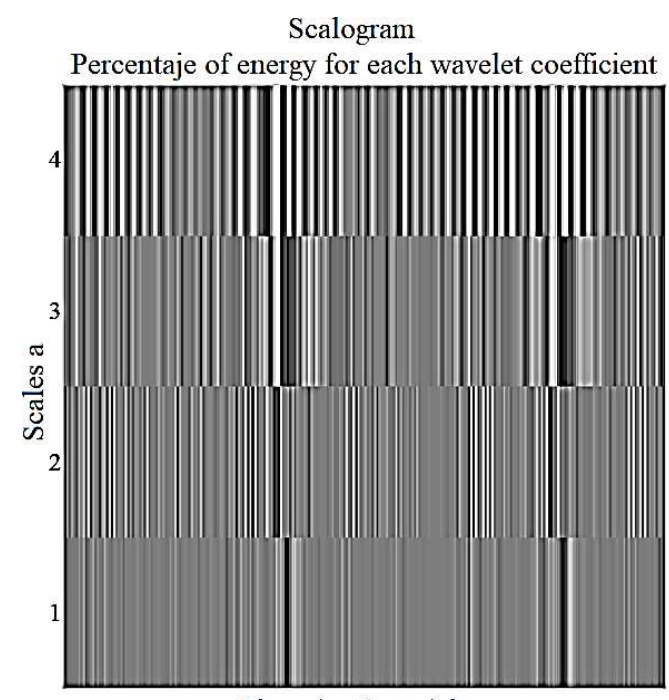

Time (or Space) b

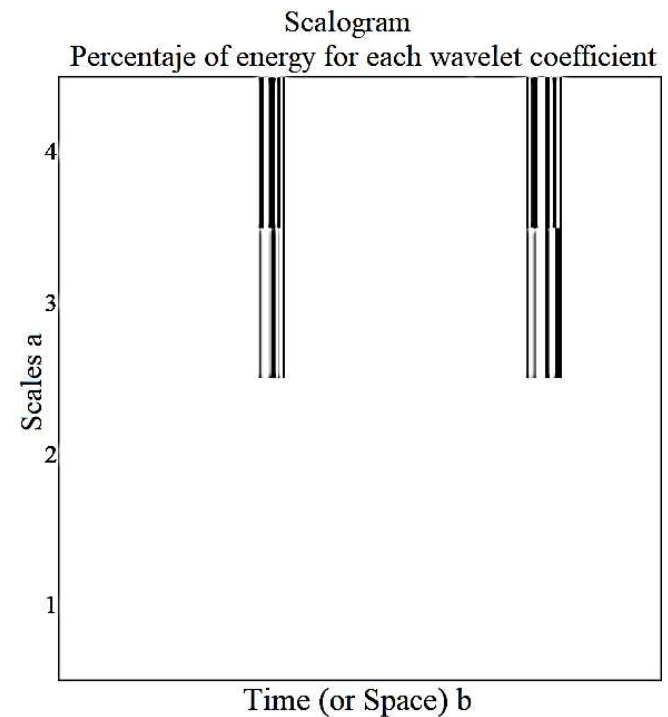

Figure 4: Graphical representation or scalogram to the left image and the thresholded wavelet coefficient matrix $T(a, b)$ to the right

As can be observed in Figure 4 there is a defined relation between the coefficients and the searched pattern. In our case, the graphical representations displayed in Figure 4 are used to support the detection of two blinking artifacts in the EEG recording of Figure 2. A threshold has been applied to the matrix of wavelet coefficients, as defined in equation (14). With this numerical relation, it is possible to determine the temporal location and duration of the pattern in the signal analysis process. In the case studied in this work, the number of samples $b$ is 
256 (analysis window size) and 4 levels of decomposition, which involves obtaining a coefficient matrix $[C]$ of $4 x 256$ elements. To improve the location of the blinking patterns, the columns of the coefficient matrix $T(a, b)$ were added as shown in equation (33).

$$
V(1,2, \ldots, n)=\sum_{a} T(a, b), \quad b=1,2, \ldots, n
$$

The vector $V$ obtained gives us the actual location in time of the artifact produced by the eye blinking. To achieve a tradeoff between data smoothing and real time analysis, a moving average of a small number of samples with a range of $10[\mathrm{~ms}]$ was applied. Applying the system shown in Figure 1 to the recorded signal displayed in Figure 2 allows overlying the dotted signal shown in Figure 5, which detects the occurrence of eye blinking and their duration.

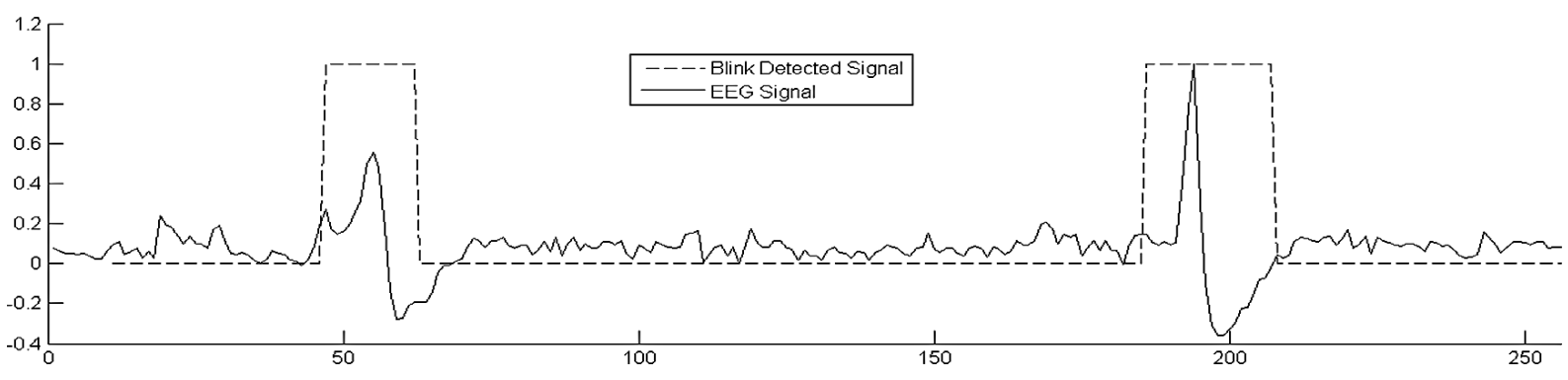

Figure 5: Overlay of original EEG signal with the dotted signal highlighting the detected artifacts and their duration produced by eye blinking
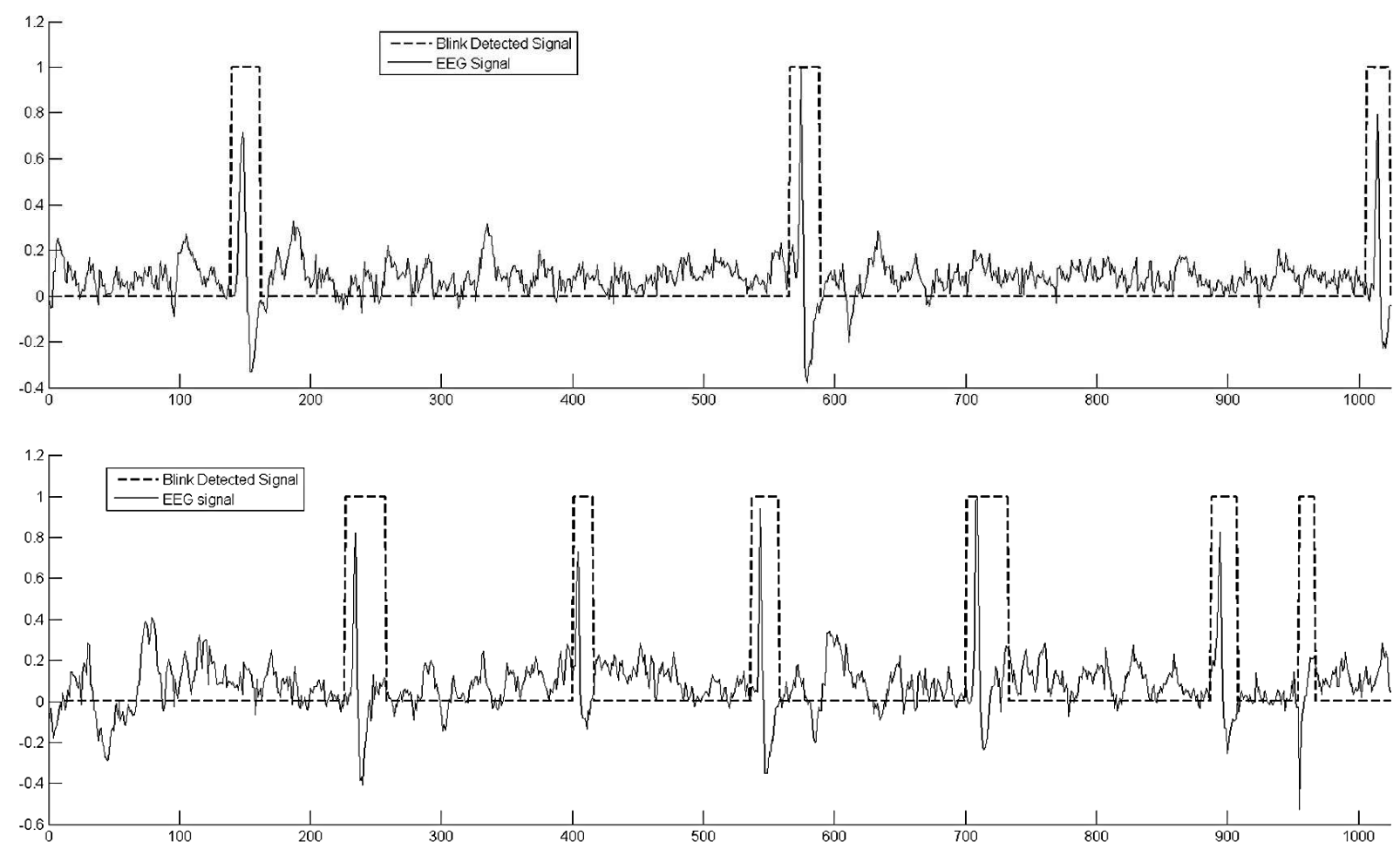

Figure 6: Examples of real-time detection of eye blinking with several analysis windows running over a $1000[\mathrm{~ms}]$ time interval. a) Top: Three eye blinks; b) Bottom: Six eye blinks 


\section{Results and discussion}

Results from this work have been tested with real-time EEG recordings introducing eyeblinking artifacts with different frequencies and amplitudes. Figure 6 shows two recordings with three and six eye blinking incidences of variable occurrences within $1000[\mathrm{~ms}]$ or one second time intervals respectively, showing variable amplitudes and duration. This figure proves that the algorithm is robust enough to filter artifacts produced by other sources that do not share similarity in shape, as well as detecting a target pattern in its many versions independent of the number of occurrences regardless of the extent and the level of symmetry in the study window.

Compared to our previous results [16] we have made some major improvements which allowed robust real-time detection in a given time interval including amplitude and duration of the eye blinking occurrences. A new mother wavelet has been designed according to the recorded patterns of the EEG waves displaying eye blinking and the new wavelet decomposition was achieved with four levels that allowed increasing the number of eye blinking artifacts within a given time analysis window demonstrated in Figures 6(a) and 6(b).

The use of a designed mother wavelet improved the global detection process of the searched pattern detection, using different observation periods for analysis, length of patterns, amplitudes and repetitions. As the relative location was obtained using the "blinkwave" transform wavelet, the thresholding and the sum of coefficients were simple and allowed quick obtaining of precise real time results.

\section{Conclusions}

The use of wavelet transforms in analyzing non-stationary signals, such as electroencephalograms, shows comparative advantages with respect to a conventional Fourier analysis. The design of a mother wavelet was the main contribution to locate the blinking artifacts in the recorded EEG signals. The paper shows in detail how to design a mother wavelet for a given case. Among the advantages of using wavelet signal processing is the multi-resolution analysis, which involves looking at multiple versions of the blinking patterns. The design of a mother wavelet used in the context of wavelet analysis incorporates features of shape, amplitude, and phase shift for detecting patterns using one-dimensional wavelet transform thus ensuring a robust detection and recognition technique of eye blinking phenomena in EEG signals. This work also contributes to bioengineering with respect to brain computer interfaces or BCI, allowing real-time detection of eye blinking. Two major applications can be visualized: improvement of current BCI devices and awareness detection systems. In addition, these eye blinking patterns, generated voluntarily by the user, can translate into simple commands for helping people with disabilities, e.g., commanding a wheelchair in forward or reverse mode. From the results of our work, we conclude that detection of the eye-blinking artifacts in EEG signals can be very helpful now that the timing information of occurrence is well defined. It should be noted that although there are alternative analyses for eye blinking detection from EEG data, those approaches operate off-line, and our method operates in real time. This approach improves respect previous work, in that there are fewer processing steps now and increased precision regarding the timing of the eye blinking events.

\section{Acknowledgment}

All authors like to thank the continuous support of the VRAC of the Technologic Metropolitan University of Chile and VRIDEI of the University of Santiago of Chile. 


\section{Author contributions}

The authors contributed equally to this work.

\section{Conflict of interest}

The authors declare no conflict of interest.

\section{Bibliography}

[1] Aeschbach, D.; Borbely, A. (1993). All-night dynamics of the human sleep EEG, Journal of Sleep Research, 2, 70-81, 1993.

[2] Bayliss, J. (2001). Flexible Brain Computer Interface, PhD thesis, Comp. Science Dept., University of Rochester, 2001.

[3] Binnie, C.; Cooper, R.; Maguire, F.; Osselton, J.; Prior, P.; Tedman, B.(2003). Clinical Neurophysiology, Elsevier Academic Press, 2003.

[4] Buzsaki, G. (2006). Rhythms of the Brain; Oxford University Press, Inc., ISBN-13 978-019-530106-9, 2006.

[5] Chambayil, B.; Singla, R.; Jha, R. (2010). EEG Eye Blink Classification Using Neural Network, Proceedings of the World Congress on Engineering, Vol I. WCE 2010, London, U.K. 2010

[6] Daubechies, I. (1992). Ten Lectures on Wavelets (CBMS-NSF Regional Conference Series in Applied Mathematics), Society for Industrial \& Applied Mathematics, U.S. 1992.

[7] Dzitac, I.; Vesselenyi, T.; Tarca, R.C. (2011). Identification of ERD using Fuzzy Inference Systems for Brain-Computer Interface, International Journal of Computers Communications \& Control, 6(3), 403-417, 2011.

[8] Fisch B.J. (1999). EEG PRIMER Basic Principles of Digital and Analog EEG. 3rd Edition, Elsevier Academic Press, 1999.

[9] Gilmore R.L. (1994). American electroencephalographic society guidelines in electroencephalography, evoked potentials, and polysomnography, Journal of Clinical Neurophysiology, 11, 147, 1994.

[10] Gloor P. (1969). Hans Berger on the Electroencephalogram of Man. Amsterdam, Elsevier Publishing Company, 1969.

[11] Mallat, S. (1989). A theory for multiresolution signal decomposition: the wavelet representation, IEEE Transactions on Pattern Analysis and Machine Intelligence, 11(7), 674-693, 1989.

[12] Mallat, S. (2009). A Wavelet Tour of Signal Processing, The Sparse Way. 3rd Ed., Library of Congress Cataloging-in-Publication. Data, Elsevier Inc. Burlington, EEUU, 205-206, 2009.

[13] Neurosky Inc. (2016). BCI: Entertainment's Brain Hacking Tool for Control \& Monitoring, http://neurosky.com/wp-content/uploads/2016/06/Control-vs-Monitor.pdf 
[14] Misiti, M.; Misiti, Y.; Oppenheim, G.; Poggi, J.M. (2007); Wavelets and Their Applications, ISTE Ltd., 115-131, 2007.

[15] Polkko J. (2007). A Method for Detecting Eye Blinks from Single-Channel Biopotential Signal in the Intensive Care, Unit. Master's Thesis, 2007.

[16] Salinas R., E. Schachter and M. Miranda. (2012). Recognition and Real-Time Detection of Blinking Eyes on Electroencephalographic Signals Using Wavelet Transform, Lecture Notes in Computer Science, 7441, 682-690, 2012.

[17] Senthil Kumar, P.; Arumuganathan, R.; Sivakumar, K.; Vimal, C. (2008). Removal of Ocular Artifacts in the EEG through Wavelet Transform without using an EOG Reference Channel, International Journal of Open Problems in Computer Science and Mathematics, 1, 188-200, 2008.

[18] Sharbroug, F.; Chatrian, G.E.; Lesser,R.P.; Luders, H.; Nuwer, M.; Picton, T.W. (1991). American Electroencephalographic Society Guidelines for Standard Electrode Position Nomenclature. Journal of Clinical Neurophysiology, 8, 200-202, 1991. 